\title{
Fish assemblages in habitats dominated by Caulerpa taxifolia and native seagrasses in south-eastern Australia
}

\author{
P. H. York ${ }^{1, *}$, D. J. Booth ${ }^{1}$, T. M. Glasby ${ }^{2}$, B. C. Pease ${ }^{2}$ \\ ${ }^{1}$ Department of Environmental Sciences, University of Technology, Sydney, PO Box 123, Broadway 2007, Australia \\ ${ }^{2}$ New South Wales Department of Primary Industries, Port Stephens Fisheries Centre, Private Bag 1, Nelson Bay 2315, \\ Australia
}

\begin{abstract}
Seagrass beds in estuaries are important habitats and nursery grounds for a great variety of fishes, including many economically important species. The introduction of the invasive green alga Caulerpa taxifolia could potentially threaten the seagrasses of south-eastern Australia. This study examined the implications of the spread of $C$. taxifolia on ichthyofauna in 2 estuaries in central New South Wales. Fish assemblages were compared among adjacent habitats of $C$. taxifolia and 2 seagrass species (Posidonia australis and Zostera capricorni). Fish were sampled using a small beam trawl to test for differences among habitats in (1) the species composition of the fish assemblages, (2) total abundance and species richness of fishes, and (3) abundances of major fish families. Fish assemblages separated into 3 significantly distinct groupings based on habitat. Total abundances of fishes were similar among habitats; however, species richness was lower in C. taxifolia. The fish assemblages in C. taxifolia were largely characterised by high abundances of gobiid fishes, similar to those in Z. capricorni, and few or no syngnathid and monacanthid species when compared to seagrass fish assemblages. This suggests that if $C$. taxifolia competitively replaces native seagrass beds in the estuaries of New South Wales, the resulting change in habitat may also cause a change in fish assemblages. This could reduce the abundances of some protected and economically important fish species but may also increase abundances of other opportunistic fishes.
\end{abstract}

KEY WORDS: Caulerpa taxifolia $\cdot$ Fish assemblages $\cdot$ Seagrass $\cdot$ South-eastern Australia

\section{INTRODUCTION}

Estuaries have been shown to support a high abundance and diversity of fishes and are important nursery grounds for many species (Claridge et al. 1986, Bell \& Pollard 1989, Kennish 1990, Potter \& Hyndes 1999). Within estuaries, seagrass beds are the most significant habitat for many of these fish species including a large number with commercial and recreational importance (Pollard 1976, 1984, Bell \& Pollard 1989). Studies have shown that areas vegetated by seagrasses have a consistently higher diversity and abundance of fish when compared to adjacent unvegetated areas (Ferrell \& Bell 1991, Gray et al. 1996, Jenkins \& Wheatley 1998).
Worthington et al. (1992a) demonstrated that total fish abundance was largely a response to the presence of shelter.

The 2 dominant seagrasses in south-eastern Australia are Posidonia australis and Zostera capricorni (West et al. 1985). Several studies have shown that differences in fish assemblages exist between these seagrass species. A study of small fish sampled in adjacent beds of $Z$. capricorni and $P$. australis by Young (1981) found significant differences in species richness and the relative abundance of species between the 2 habitats in Port Hacking, New South Wales. A more comprehensive study in Botany Bay, New South Wales (Middleton et al. 1984), indicates that the differences 
in the fish assemblages are due mainly to temporal and size differences of many species, suggesting that fish recruit first to $Z$. capricorni and then move into $P$. australis when they are older. Recently, Rotherham \& West (2002) found significant differences in abundances and lengths of economically important fish species, and also the composition of fish assemblages between seagrass species in Botany Bay and Port Hacking, but not between these habitats in Sussex Inlet (200 km south). They suggest that local recruitment events and differences in the geomorphology of estuaries might also be important in determining the structure of fish assemblages.

Recently, concerns have been raised about seagrass meadows in south-eastern Australia in relation to the spread of the introduced green alga Caulerpa taxifolia. This alga was first discovered in estuaries in central New South Wales in 2000 (Schaffelke et al. 2002, Glasby et al. 2005). C. taxifolia has reportedly caused problems in the Mediterranean Sea, where it has spread rapidly since its first discovery in 1984 (Meinesz et al. 2001). Studies in the Mediterranean show that Posidonia oceanica seagrass beds have a positive effect on the growth of $C$. taxifolia and that sparse beds of the seagrass are particularly vulnerable to invasion (Ceccherelli \& Cinelli 1999). Remote sensing and SCUBA surveys in Menton Bay and along $44 \%$ of the coast of France suggest, however, that invasion by $C$. taxifolia has not caused a significant reduction in the abundance of Posidonia oceanica (Jaubert et al. 1999, 2003). In Australia it is yet to be established whether $C$. taxifolia is having a negative impact on seagrasses; however, it has been observed growing adjacent to and in seagrass beds, as well as in previously unvegetated sediments (Glasby et al. 2005).

Few published studies have compared fish assemblages between native seagrasses and Caulerpa taxifolia. One such study on the French coast compared assemblages in areas colonised by $C$. taxifolia with those in native Posidonia oceanica beds (Francour et al. 1995). They found similarities in species richness and the composition of fish assemblages, but lower abundance and biomass in the site invaded by C. taxifolia. In the Ligurian Sea, Italy, areas colonised by C. taxifolia had higher species richness and almost double the abundances of fish compared to the native seagrass Cymodocea nodosa (Relini et al. 1998). The results of both these studies should, however, be treated cautiously because they lacked spatial replication, comparing only one site of invasion to one reference site.

The present study compared fish assemblages in 2 native seagrasses, the strapweed Posidonia australis and the eelgrass Zostera capricorni, with those found in adjacent areas colonised by Caulerpa taxifolia, in 2 south-eastern Australian estuaries. The aims of the study were to determine if fish communities in native seagrass beds differed from those in areas colonised by C. taxifolia with respect to (1) composition of fish assemblages, (2) total abundance and species richness of fishes and (3) abundances of the families of fishes that were dominant in the assemblages throughout the study.

\section{MATERIALS AND METHODS}

Study areas. The study was carried out at 3 sites in south-eastern Australia (Fig. 1). Two sites were sampled within Botany Bay, a large, marine-dominated embayment in the southern suburbs of Sydney $\left(34^{\circ} 00^{\prime} \mathrm{S}, 151^{\circ} 14^{\prime} \mathrm{E}\right)$. Much of the land use in the catchment of the bay is urban and industrial. These 2 sites were located at Bona Point and Quibray Bay, on the southern side of the bay within the Towra Point aquatic reserve where all forms of fishing are banned.

The third site was situated in Port Hacking $\left(34^{\circ} 05^{\prime} \mathrm{S}\right.$, $\left.151^{\circ} 09^{\prime} \mathrm{E}\right)$, about $10 \mathrm{~km}$ south of Botany Bay. Port Hacking is a drowned river valley that is bordered on its northern side by residential suburbs and on its southern shore by National Park. It is a relatively pristine waterway, as much of its catchment is situated in national park land, and there is a lack of industry on its foreshores (Anonymous 1997). Port Hacking has been

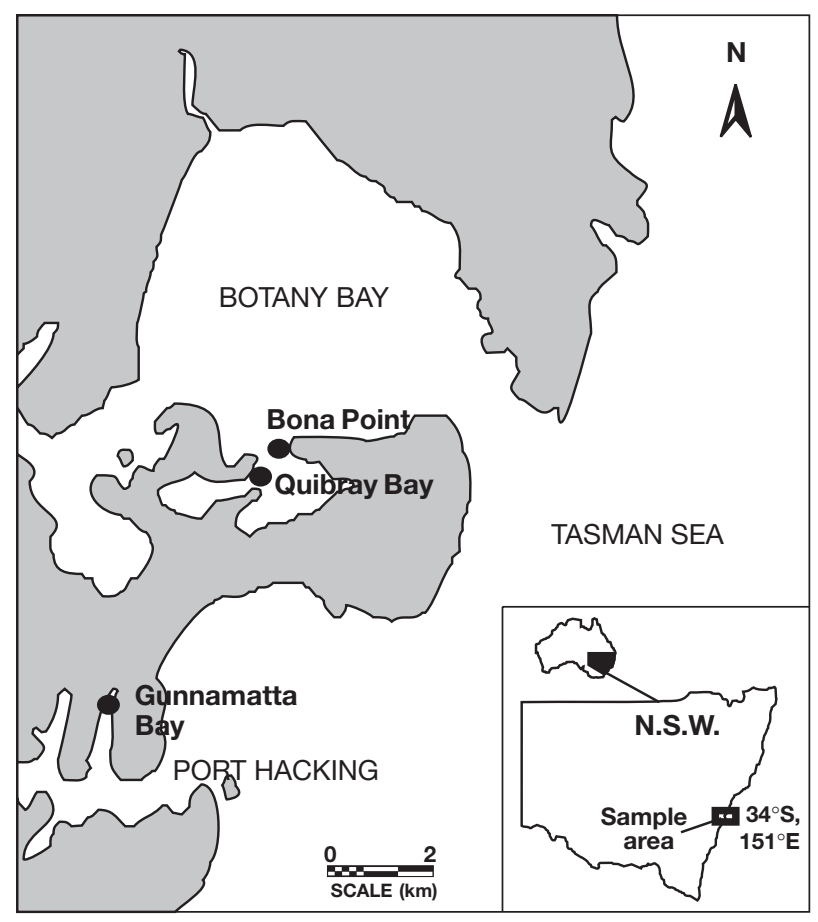

Fig. 1. Location of study sites in south-eastern Australia 
closed to commercial fishing since 1886 (Pease \& Herbert 2002) but has long been a popular recreational fishing spot in southern Sydney (Henry et al. 1987). This site was located at the head of Gunnamatta Bay. Port Hacking was one of the first estuaries in southeastern Australia where Caulerpa taxifolia was discovered (Schaffelke et al. 2002). Due to the differences in vegetation distributions within estuaries, depth varied among habitats at all sites with Zostera capricorni at an average depth of $0.5 \mathrm{~m}$, Posidonia australis at between 1 and $1.5 \mathrm{~m}$ and $C$. taxifolia at around $1.5 \mathrm{~m}$ below mean low tide.

Experimental design. In each of the 3 study sites adjacent beds of Zostera capricorni, Posidonia australis and Caulerpa taxifolia were present and separated by no more than $300 \mathrm{~m}$. Individual habitats ranged from ca. $200 \mathrm{~m}^{2}$ of Z. Capricorni at Gunnamatta Bay to greater than 2 ha of $P$. australis in Quibray Bay. Fish assemblages in each benthic habitat were sampled using a small beam trawl, $1 \mathrm{~m}$ wide $\times 0.5 \mathrm{~m}$ high, with $3 \mathrm{~mm}$ mesh size. Replicate hauls (4 per habitat) of premeasured $25 \mathrm{~m}$ lengths were carried out at each site by towing the beam trawl behind a small boat at a speed of 3 to $5 \mathrm{~km} \mathrm{~h}^{-1}$. Thus the area sampled was $25 \mathrm{~m}^{2}$ per haul. Beam trawls have been used extensively for catching juvenile fish (Young 1981, Bell et al. 1992, Ferrell et al. 1993). Although seine nets have been found to be more effective than beam trawls for determining the relative proportion of species and estimating densities of fishes in seagrass (Guest et al. 2003), it was not practical to use a seine net in this study as the water was too deep at some of the sites. Sampling was done at roughly monthly intervals from early September 2003 to early February 2004, the main period of recruitment for economically important fish species (McNeill et al. 1992).

Fish caught in the beam trawl were identified, counted and then returned to the water. The vast majority of fish that were released swam away freely and without signs of ill effects. A single specimen of any species that could not be identified in the field was placed in a clove oil solution until death, then kept on ice and taken back to the laboratory for identification to species based on Kuiter (2000) and Gommon et al. (1994).

Previous studies have shown that tidal phase (Kingsford \& Suthers 1996), time of day (Griffiths 2001) and moon phase (Smith \& Suthers 2000) can affect fish abundances in seagrasses. In this study, sampling was carried out randomly with respect to tidal phase, but shallow water depths prevented sampling of Zostera capricorni at low tide. Although samples were collected in daytime only, time of day was randomised and sampling was focused around the full moon where possible to allow for temporal variation in recruitment and abundance of fishes that may be influenced by these factors. Salinity and water temperature were also recorded in each habitat on each sampling day using a multi-probe YSI meter to monitor for temporal and special fluctuations likely to affect the abundances of estuarine fishes.

Data analyses. The similarity of fish communities among sites and habitats for each of the 4 sampling periods was examined using non-parametric multivariate techniques (PRIMER package v5). Total abundances $\left(25 \mathrm{~m}^{-2}\right)$ for each species in each sampling period were square root transformed and a Bray-Curtis similarity matrix was created (Clarke \& Warwick 2001). The results were then plotted on a 2D scaling (MDS) plot. Analyses of similarity (ANOSIM) were conducted using a 2-way crossed design with site and habitat as the factors determined a priori. Where these groups were significantly different, similarity percentages (SIMPER) were calculated using square root transformed, unstandardised data to determine which species made the largest contribution to the dissimilarities (Clarke \& Warwick 2001).

Total abundance was expressed as the number of fish in each $25 \mathrm{~m}^{2}$ sample. Species richness was estimated for $25 \mathrm{~m}^{2}$ using the equation:

$$
S_{\text {tot }}=S_{\text {obs }}+\left(a^{2} / 2 b\right)
$$

where $S_{\text {tot }}$ is the predicted total species richness, $S_{\text {obs }}$ is the observed number of species at a given sampling effort, $a$ is the number of species represented by a single individual, and $b$ is the number of species represented by exactly 2 individuals (Chao 1984). This extrapolative technique was found to be the best for limited sampling effort (Foggo et al. 2003). Analysis of variance (ANOVA) tests were applied to the total abundance and species richness indices as well as the abundances of the 3 major families represented in the study (Sygnathidae, Monacanthidae and Gobidae). Homogeneity of variance was not satisfied (Cochran's test), so the data were transformed $[\ln (x+1)]$ to fulfil this assumption. A 3-factor ANOVA model was used, with habitat as a fixed variable and site and time as random variables, meaning that an $F$-test for habitat could not be calculated unless either the Habitat $\times$ Site or Habitat $\times$ Time interaction could be eliminated. Student-Newman-Keul (SNK) tests were used to compare means when significant differences were detected by ANOVA.

\section{RESULTS}

The abundance of each species and their percentage contribution to the total catch in each habitat is summarised in Table 1. During the study 2744 individuals 
Table 1. List of fish species caught during the study showing their abundance $\left(1.2 \mathrm{~km}^{2}\right.$ area) and percentage contribution among the 3 habitats. Total abundance $(\mathrm{N})$ of each fish species during the study $\left(3.6 \mathrm{~km}^{2}\right.$ area) and their percentage contribution within the study are also shown. Z: Zostera capricorni, P: Posidonia australis, C: Caulerpa taxifolia

\begin{tabular}{|c|c|c|c|c|c|c|c|c|c|}
\hline \multirow{2}{*}{ Family and species } & \multirow{2}{*}{ Common name } & \multicolumn{3}{|c|}{ Abundance/habitat } & \multicolumn{3}{|c|}{$\%$ contribution } & \multicolumn{2}{|l|}{ Total } \\
\hline & & Z & $\mathrm{P}$ & $\mathrm{C}$ & $\mathrm{Z}$ & $\mathrm{P}$ & C & $\mathrm{N}$ & $\%$ \\
\hline Syngnathidae & Pipefishes and Seahorses & 425 & 93 & 11 & 32.3 & 14.5 & 1.4 & 529 & 19.3 \\
\hline Hippocampus whitei & White's seahorse & 1 & 3 & 1 & 0.1 & 0.5 & 0.1 & 5 & 0.2 \\
\hline Stigmatapora argus & Spotted pipefish & 32 & 69 & 0 & 2.4 & 10.8 & 0 & 101 & 3.7 \\
\hline Stigmatapora nigra & Wide-bodied pipefish & 87 & 3 & 0 & 6.6 & 0.5 & 0 & 90 & 3.3 \\
\hline Urocampus carinirostris & Hairy pipefish & 303 & 10 & 8 & 23.0 & 1.6 & 1.0 & 321 & 11.7 \\
\hline Vanacampus margaritifer & Mother-of-pearl pipefish & 2 & 8 & 2 & 0.2 & 1.2 & 0.3 & 12 & 0.4 \\
\hline Scorpaenidae & Scorpionfishes & & & & & & & & \\
\hline Centropogon australis & Common fortescue & 76 & 88 & 90 & 5.8 & 3.7 & 1.5 & 254 & 9.3 \\
\hline Ambassidae & Glassfishes & & & & & & & & \\
\hline Ambassis jacksoniensis & Port Jackson perchlet & 7 & 20 & 54 & 0.5 & 3.1 & 6.9 & 81 & 3.0 \\
\hline Terapontidae & Grunters & & & & & & & & \\
\hline Pelates sexlineatus & Six-lined trumpeter & 25 & 23 & 7 & 1.9 & 3.6 & 0.9 & 55 & 2.0 \\
\hline Apogonidae & Cardinalfishes & & & & & & & & \\
\hline Siphamia cephalotes & Wood's siphonfish & 1 & 74 & 71 & 0.1 & 11.5 & 9.0 & 146 & 5.3 \\
\hline Gerreidae & Silver bellies & & & & & & & & \\
\hline Gerres subfasciatus & Common silver belly & 0 & 1 & 0 & 0 & 0.2 & 0 & 1 & 0.1 \\
\hline Sparidae & Snapper and Bream & 37 & 0 & 0 & 2.8 & 0 & 0 & 37 & 1.3 \\
\hline Acanthopagrus australis & Yellow-fin bream & 2 & 0 & 0 & 0.2 & 0 & 0 & 2 & 0.1 \\
\hline Rhabdosargus sarba & Tarwhine & 35 & 0 & 0 & 2.7 & 0 & 0 & 35 & 1.3 \\
\hline Girellidae & Blackfishes & & & & & & & & \\
\hline Girella tricuspidata & Luderick & 23 & 1 & 0 & 1.8 & 0.2 & 0 & 24 & 0.9 \\
\hline Enoplosidae & Oldwives & & & & & & & & \\
\hline Enoplosus armatus & Old wife & 2 & 2 & 0 & 0.2 & 0.3 & 0 & 4 & 0.1 \\
\hline Labridae & Wrasses & & & & & & & & \\
\hline Achoerodus viridis & Eastern blue groper & 9 & 5 & 0 & 0.7 & 0.8 & 0 & 14 & 0.5 \\
\hline Odacidae & Weed-whitings & & & & & & & & \\
\hline Neoodax balteatus & Little rock-whiting & 1 & 46 & 11 & 0.1 & 7.2 & 1.4 & 58 & 2.1 \\
\hline Scaridae & Parrotfishes & & & & & & & & \\
\hline Leptoscarus vaigiensis & Seagrass parrotfish & 1 & 0 & 0 & 0.1 & 0 & 0 & 1 & 0.1 \\
\hline Blennidae & Blennies & & & & & & & & \\
\hline Petroscirtes lupus & Sabre-toothed blenny & 0 & 0 & 1 & 0 & 0 & 0.1 & 1 & 0.1 \\
\hline Clinidae & Weedfishes & & & & & & & & \\
\hline Cristiceps australis & Crested weedfish & 11 & 42 & 12 & 0.8 & 6.6 & 1.5 & 65 & 2.4 \\
\hline Gobiidae & Gobies & 600 & 67 & 518 & 45.6 & 10.3 & 65.9 & 1184 & 43.2 \\
\hline Arenigobius bifrenatus & Bridled goby & 1 & 0 & 5 & 0.1 & 0 & 0.6 & 6 & 0.2 \\
\hline Arenigobius frenatus & Half-bridled goby & 177 & 21 & 138 & 13.4 & 3.3 & 17.6 & 336 & 12.2 \\
\hline Bathygobius kreffti & Frayed-fin goby & 52 & 22 & 65 & 4.0 & 3.4 & 8.3 & 139 & 5.1 \\
\hline Cristagobius gobioides & Oyster goby & 0 & 0 & 2 & 0 & 0 & 0.3 & 2 & 0.1 \\
\hline Favonigobius exquisitus & Exquisite sand-goby & 24 & 4 & 48 & 1.8 & 0.6 & 6.1 & 76 & 2.8 \\
\hline Favonigobius lateralis & Long-finned goby & 184 & 8 & 39 & 14.0 & 1.3 & 5.0 & 231 & 8.4 \\
\hline Gobiopterus semivestita & Glass goby & 2 & 0 & 0 & 0.2 & 0 & 0 & 2 & 0.1 \\
\hline Redigobius macrostoma & Large-mouthed goby & 160 & 11 & 221 & 12.2 & 1.7 & 28.1 & 392 & 14.3 \\
\hline Bothidae & Flounders & & & & & & & & \\
\hline Pseudorhombus jenynsii & & 0 & 1 & 1 & 0 & 0.2 & 0.1 & 2 & 0.1 \\
\hline Monacanthidae & Leatherjackets & 97 & 177 & 8 & 7.4 & 27.6 & 1.3 & 284 & 10.4 \\
\hline Acanthaluteres spilomelanurus & Bridled leatherjacket & 15 & 51 & 3 & 1.1 & 8.0 & 0.4 & 69 & 2.5 \\
\hline Brachaluteres jacksonianus & Pygmy leatherjacket & 0 & 8 & 0 & 0 & 1.3 & 0 & 8 & 0.3 \\
\hline Meuschenia freycineti & Six-spined leatherjacket & 62 & 77 & 3 & 4.7 & 12.0 & 0.4 & 142 & 5.2 \\
\hline Meuschenia trachylepis & Yellow-finned leatherjacket & 10 & 16 & 0 & 0.8 & 2.5 & 0 & 26 & 1.0 \\
\hline Monacanthus chinensis & Fan-belly leatherjacket & 9 & 11 & 4 & 0.7 & 1.7 & 0.5 & 24 & 0.9 \\
\hline Nelusetta ayraudi & Chinaman leatherjacket & 1 & 6 & 0 & 0.1 & 0.9 & 0 & 7 & 0.3 \\
\hline Scobinichthys granulatus & Rough leatherjacket & 0 & 8 & 0 & 0 & 1.3 & 0 & 8 & 0.3 \\
\hline Tetradontidae & Toadfishes & & & & & & & & \\
\hline Tetractenos glaber & Smooth toadfish & 2 & 1 & 0 & 0.2 & 0.2 & 0 & 3 & 0.1 \\
\hline Diodontidae & Porcupinefishes & & & & & & & & \\
\hline Dicotylichthys punctulatus & Three-bar porcupinefish & 0 & 1 & 0 & 0 & 0.2 & 0 & 1 & 0.1 \\
\hline
\end{tabular}


were caught, representing 37 species and 19 families. Zostera capricorni had the highest abundance with 1317 individuals belonging to 30 species and 15 families, followed by Caulerpa, with 786 individuals from 21 species and 11 families, and Posidonia australis with 641 individuals belonging to 30 species from 16 families. The most abundant species were from Family Gobiidae (gobies) and included Redigobius macrostoma, Arenigobius frenatus, Favonigobius lateralis and Bathygobius kreffti, which were distributed across all habitats. Family Syngnathidae (pipefishes and seahorses) comprised a large portion of the catch with Urocampus carinirostris and Stigmatapora nigra mainly inhabiting Z. capricorni beds and Stigmatapora argus mainly in $P$. australis beds. Monacanthidae (leatherjackets) such as Meuschenia freycineti and Acanthaluteres spilomelanurus were also relatively abundant in the seagrass habitats. Other fish caught in relatively large numbers were 2 pelagic schooling species Siphamia cephalotes and Ambassis jacksoniensis as well as the benthic species Centropogon australis, Neoodax balteatus and Cristiceps australis. Recruits of economically important species Rhabdosargus sarba, Acanthopagrus australis and Girella tricuspidata were found infrequently and in low numbers during the study, but when caught they were almost exclusively in $Z$. capricorni beds.

Fish assemblages in the 3 habitats showed reasonably consistent patterns during all 4 sampling periods based on the MDS plots (Fig. 2). The samples grouped together based on habitat and were partially separated from one another. Overall, Caulerpa taxifolia and Posidonia australis appear to be the most distant in terms of assemblages with Zostera capricorni assemblages lying between the two. Samples within C. taxifolia in October and January and Z. Capricorni in November/December and January showed greater spread in relation to each other indicating greater variation within these habitats than the other months. The stress values for the 4 different multidimensional scaling plots ranged between
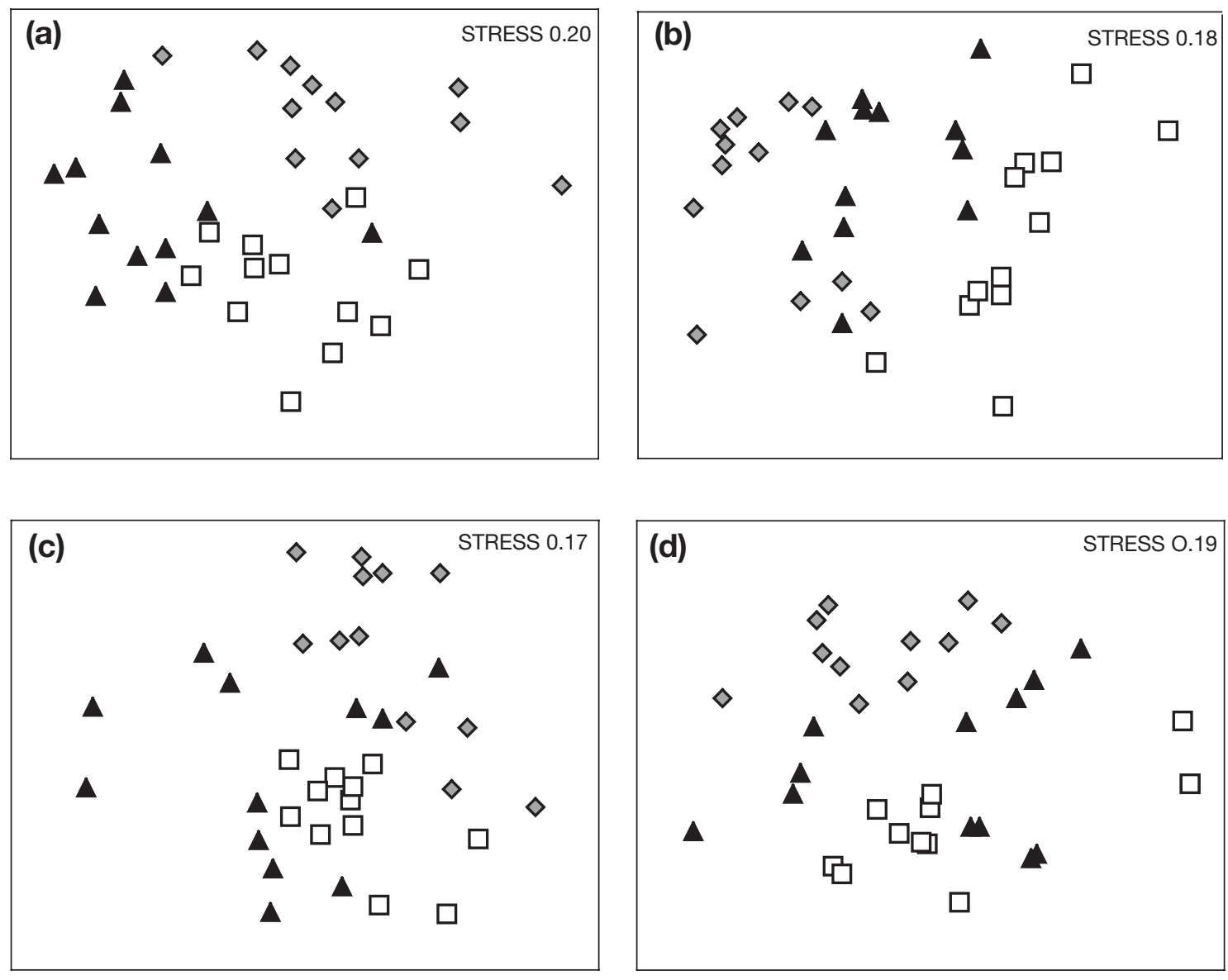

Fig. 2. Multidimensional scaling plots based on square root transformed abundance data for the 4 sampling periods: (a) September 2003, (b) October 2003, (c) November/December 2003, (d) January 2004. A: Zostera capricorni, $\diamond:$ Posidonia australis, $\square$ : Caulerpa taxifolia 
Table 2. ANOSIM using a 2-factor crossed design (habitat and site differences) at each of the 4 sampling periods ( $\mathrm{S}=\mathrm{Sep}$ tember 2003, O = October 2003, N/D = November/December 2003, J = January 2004). Global R values calculated after 5000 random permutations. All values significant at $p<0.01$

\begin{tabular}{|lcccc|}
\hline & $\mathrm{S}$ & $\mathrm{O}$ & $\mathrm{N} / \mathrm{D}$ & $\mathrm{J}$ \\
\hline Habitat & 0.686 & 0.664 & 0.601 & 0.736 \\
Site & 0.471 & 0.573 & 0.431 & 0.572 \\
\hline
\end{tabular}

Table 3. Pairwise comparisons of differences between habitats (ANOSIM) at each of the 4 sampling periods (see Table 2). Global $\mathrm{R}$ values and significance levels were calculated after 5000 random permutations. ${ }^{*}$ Significant results at $\mathrm{p}<0.05$

\begin{tabular}{|lrrrrrr|}
\hline & $\begin{array}{c}\text { Z. capricorni vs. } \\
\text { P. australis } \\
\text { Global R }\end{array}$ & \multicolumn{2}{c}{$\begin{array}{c}\text { Z. capricorni vs. } \\
\text { C. taxifolia }\end{array}$} & \multicolumn{2}{c|}{$\begin{array}{c}\text { P. australis vs. } \\
\text { C. taxifolia }\end{array}$} \\
& Global R & p & Global R & p \\
\hline $\mathrm{S}$ & 0.809 & $>0.01^{*}$ & 0.609 & $>0.01^{*}$ & 0.764 & $>0.01^{*}$ \\
$\mathrm{O}$ & 0.910 & $>0.01^{*}$ & 0.712 & $>0.01^{*}$ & 0.549 & $>0.01^{*}$ \\
$\mathrm{~N} / \mathrm{D}$ & 0.764 & $0.01^{*}$ & 0.635 & $>0.01^{*}$ & 0.684 & $>0.01^{*}$ \\
$\mathrm{~J}$ & 0.705 & $>0.01^{*}$ & 0.767 & $>0.01^{*}$ & 0.868 & $>0.01^{*}$ \\
\hline
\end{tabular}

0.17 and 0.2 , which are high but acceptable for display on a 2D plot (Clarke 1993).

The analysis of similarity (ANOSIM) for each sampling period (Table 2) showed that there were significant differences in the fish assemblages at all times among all habitats and sites. In all cases these differences were stronger between habitats than they were between sites, as shown by the global $\mathrm{R}$ values that ranged from 0.601 to 0.736 for habitats compared to 0.572 to 0.431 for sites. Pairwise comparisons (Table 3) showed significant differences between all habitats at every sampling period.

The application of SIMPER analysis (Table 4) showed that differences between the 2 seagrass habitats were due largely to greater abundances of Urocampus carinirostris, Arenigobius frenatus and Favonigobius lateralis in Zostera capricorni beds and a larger contribution of Stigmatapora argus, Siphamia cephalotes and Meuschenia freycineti in Posidonia australis beds. Assemblages in Caulerpa taxifolia differed from those in $Z$. capricorni due to the high abundance of $U$. carinirostris in $Z$. capricorni and its absence in C. taxifolia as well as the greater abundances of Redigobius

Table 4. SIMPER analysis showing the percentage contribution (\% C) of the 5 most important fish species to the dissimilarity of samples between different habitats (pairwise comparisons) for the 4 sampling periods. Also shown are the average abundances (\#) $25 \mathrm{~m}^{-2}$ for these species in each habitat. $\mathrm{Z}=$ Zostera capricorni, $\mathrm{P}=$ Posidonia australis, $\mathrm{C}=$ Caulerpa taxifolia. See Table 1 for full species names. $\mathrm{AD}=$ average dissimilarity

\begin{tabular}{|c|c|c|c|c|c|c|c|c|c|c|c|}
\hline \multicolumn{4}{|c|}{ Z. capricorni vs. P. australis } & \multicolumn{4}{|c|}{ Z. capricorni vs. C. taxifolia } & \multicolumn{4}{|c|}{ P. australis vs. C. taxifolia } \\
\hline & $\# \mathbf{Z}$ & \# $\mathbf{P}$ & $\% \mathbf{C}$ & & $\# \mathbf{Z}$ & \# C & $\% \mathrm{C}$ & & \# $\mathbf{P}$ & \# C & $\% \mathrm{C}$ \\
\hline \multicolumn{12}{|l|}{ September 2003} \\
\hline F. lateralis & 4.4 & 0.4 & 10.0 & R. macrostoma & 1.08 & 8.42 & 15.2 & R. macrostoma & 0.2 & 8.4 & 13.1 \\
\hline U. carinirostris & 4.3 & 0.3 & 9.2 & A. frenatus & 4.42 & 2.75 & 12.5 & A. spilomelanurus & 3.2 & 0.3 & 9.9 \\
\hline A. spilomelanurus & 0.5 & 3.2 & 7.9 & U. carinirostris & 4.25 & 0.50 & 12.3 & A. frenatus & 0.6 & 2.8 & 8.6 \\
\hline A. frenatus & 4.4 & 0.6 & 7.6 & F. lateralis & 4.42 & 1.17 & 11.1 & S. cephalotes & 1.9 & 0.0 & 8.0 \\
\hline S. cephalotes & 0.0 & 1.9 & 7.2 & S. nigra & 2.33 & 0.00 & 8.3 & S. argus & 2.1 & 0.0 & 6.6 \\
\hline \multicolumn{12}{|l|}{ October 2003} \\
\hline $\mathrm{AD}=71.45$ & & & & $\mathrm{AD}=\mathbf{7 5 . 0 8}$ & & & & $\mathrm{AD}=81.67$ & & & \\
\hline F. lateralis & 8.6 & 0.2 & 12.6 & U. carinirostris & 6.33 & 0.08 & 13.1 & M. freycineti & 2.7 & 0.1 & 11.4 \\
\hline U. carinirostris & 6.3 & 0.3 & 10.6 & F. lateralis & 8.58 & 0.75 & 12.9 & A. frenatus & 0.3 & 2.9 & 10.3 \\
\hline A. frenatus & 3.7 & 0.3 & 7.2 & M. freycineti & 3.67 & 0.08 & 10.2 & R. macrostoma & 0.3 & 3.1 & 9.5 \\
\hline M. freycineti & 3.7 & 2.7 & 6.5 & R. macrostoma & 1.25 & 3.08 & 8.6 & S. cephalotes & 1.9 & 0.0 & 7.7 \\
\hline S. cephalotes & 0.0 & 1.9 & 6.2 & A. frenatus & 3.67 & 2.92 & 8.6 & C. australis & 1.2 & 1.9 & 7.1 \\
\hline \multicolumn{12}{|c|}{ November/December 2003} \\
\hline$A D=79.67$ & & & & $\mathrm{AD}=\mathbf{7 0 . 2 0}$ & & & & $\mathrm{AD}=77.49$ & & & \\
\hline A. frenatus & 3.8 & 0.4 & 10.1 & A. frenatus & 3.75 & 3.67 & 11.7 & A. frenatus & 0.4 & 3.7 & 14.6 \\
\hline U. carinirostris & 6.8 & 0.1 & 9.8 & R. macrostoma & 3.75 & 2.00 & 11.5 & S. argus & 1.6 & 0.0 & 10.0 \\
\hline S. argus & 0.2 & 1.6 & 8.5 & U. carinirostris & 6.83 & 0.00 & 10.6 & R. macrostoma & 0.1 & 2.0 & 9.8 \\
\hline F. lateralis & 1.7 & 0.1 & 7.9 & B. kreffti & 0.67 & 2.25 & 8.9 & B. kreffti & 1.0 & 2.3 & 8.9 \\
\hline S. nigra & 3.2 & 0.0 & 7.6 & F. lateralis & 1.67 & 1.17 & 8.7 & S. cephalotes & 0.7 & 5.8 & 8.9 \\
\hline \multicolumn{12}{|l|}{ January 2004} \\
\hline $\mathrm{AD}=74.88$ & & & & $\mathrm{AD}=73.66$ & & & & $\mathrm{AD}=76.63$ & & & \\
\hline U. carinirostris & 7.8 & 0.1 & 12.1 & U. carinirostris & 7.83 & 0.08 & 13.3 & M. freycineti & 2.3 & 0.1 & 11.5 \\
\hline C. australis & 2.7 & 4.0 & 9.9 & R. macrostoma & 7.25 & 4.92 & 12.5 & C. australis & 4.0 & 2.8 & 11.3 \\
\hline R. macrostoma & 7.3 & 0.3 & 8.7 & C. australis & 2.67 & 2.83 & 10.3 & F. exquisites & 0.2 & 2.8 & 10.5 \\
\hline A. frenatus & 2.9 & 0.5 & 7.6 & F. exquisites & 1.67 & 2.75 & 10.0 & R. macrostoma & 0.3 & 4.9 & 9.9 \\
\hline M. freycineti & 0.6 & 2.3 & 7.5 & A. frenatus & 2.92 & 2.17 & 8.8 & S. cephalotes & 1.7 & 0.1 & 8.4 \\
\hline
\end{tabular}


macrostoma and A. frenatus in C. taxifolia. Fish assemblages in $P$. australis differed from those in C. taxifolia due to much higher abundances of $S$. argus, $M$. freycineti and $S$. cephalotes and lower abundances of $R$. macrostoma and $A$. frenatus in the seagrass.

ANOVA determined interactions between site and habitat were significant for both total abundance of fishes and species richness (Table 5). Total abundance was similar among habitats at Gunnamatta Bay and Bona Point, but there were significantly more fishes in Zostera capricorni beds in Quibray Bay (SNK test; Fig. 3a). This was the cause of the significant Site $\times$ Habitat interaction, although there was a similar, but non-significant, trend in Gunnamatta Bay. Similarly, species richness differed among habitats at 2 sites (Fig. 3b). Caulerpa taxifolia habitats had significantly fewer species than the seagrass habitats in Gunnamatta Bay and Quibray Bay (SNK test). In contrast, for Bona Point, Posidonia australis beds had significantly more species than C. taxifolia or Z. capricorni beds.

Patterns of abundance of fishes from Family Syngnathidae among habitats differed at each site (Table 5c). Zostera capricorni had significantly higher abundances of syngnathids compared to Posidonia australis at both Gunnamatta Bay and Quibray Bay, but there were similar abundances in the 2 seagrass habitats at Bona Point (SNK test, Fig. 4a). Abundances of syngnathids in Caulerpa taxifolia were significantly less than in either seagrass habitat at all 3 sites (Fig. 4a).

Abundance of Monacanthidae also differed among habitats and sites (Table 5d). In Gunnamatta Bay and at Bona Point, significantly more monacanthids were caught in seagrass habitats than in Caulerpa taxifolia, whilst in Quibray Bay this significant difference occurred only between Zostera capricorni and C. taxifolia (SNK test; Fig. 4b). Moreover, in the 2 sites in Botany Bay (Bona Point and Quibray Bay), Posidonia australis contained significantly higher numbers of syngnathid fishes than did Z capricorni. Interestingly, there was an opposite trend in Gunnamatta Bay (Fig. 4b).

For Gobiidae, there was a significant interaction among site, habitat and time (Table 5e). Abundances of species from this family were consistently, and often significantly, lowest in Posidonia australis (SNK test; Fig. 4c). Although the magnitude of differences in abundances of gobies among habitats differed among times and sites, there was a consistent trend for gobies to be most abundant in Zostera capricorni and Caulerpa taxifolia (Fig. 4c).

Water temperature ranged from $12.7^{\circ} \mathrm{C}$ in September to $26.8^{\circ} \mathrm{C}$ in January, with an overall trend of rising temperatures between each successive sampling period. At no time, however, was there a difference greater than $2^{\circ} \mathrm{C}$ between the 3 habitats on any sampling day.
Table 5. Results of 3-factor ANOVA for total abundance, species richness and abundance of major families. Data were $\ln (x+1)$ transformed to reduce heterogeneity of variance. Significant differences at ${ }^{*} p=0.05$ and ${ }^{* *} p=0.01$. N/A: not applicable

\begin{tabular}{|c|c|c|c|c|}
\hline Source & df & MS & $F$-value & $\mathrm{p}$ \\
\hline \multicolumn{5}{|c|}{ (a) Total abundance } \\
\hline Site $(\mathrm{S})$ & 2 & 7.6115 & 8.10 & \\
\hline Habitat (H) & 2 & 3.6989 & No test & N/A \\
\hline Time $(\mathrm{T})$ & 3 & 0.0996 & 0.11 & \\
\hline $\mathrm{S} \times \mathrm{H}$ & 4 & 2.5511 & 5.08 & $*$ \\
\hline $\mathrm{S} \times \mathrm{T}$ & 6 & 0.9397 & 3.18 & $* *$ \\
\hline $\mathrm{H} \times \mathrm{T}$ & 6 & 0.8107 & 1.62 & \\
\hline $\mathrm{S} \times \mathrm{H} \times \mathrm{T}$ & 12 & 0.5019 & 1.70 & \\
\hline Residual & 108 & 0.2956 & & \\
\hline \multicolumn{5}{|c|}{ (b) Estimated species richness } \\
\hline Site $(\mathrm{S})$ & 2 & 0.5601 & 1.41 & \\
\hline Habitat (H) & 2 & 3.4384 & No test & N/A \\
\hline Time $(\mathrm{T})$ & 3 & 0.7135 & 1.80 & \\
\hline $\mathrm{S} \times \mathrm{H}$ & 4 & 0.8036 & 4.71 & * \\
\hline $\mathrm{S} \times \mathrm{T}$ & 6 & 0.3969 & 1.68 & \\
\hline $\mathrm{H} \times \mathrm{T}$ & 6 & 0.2547 & 1.49 & \\
\hline $\mathrm{S} \times \mathrm{H} \times \mathrm{T}$ & 12 & 0.1706 & 0.72 & \\
\hline Residual & 108 & 0.2359 & & \\
\hline \multicolumn{5}{|c|}{ (c) Abundance, Syngnathidae } \\
\hline Site $(\mathrm{S})$ & 2 & 4.2853 & 12.68 & $* *$ \\
\hline Habitat $(\mathrm{H})$ & 2 & 20.0503 & No test & N/A \\
\hline Time $(\mathrm{T})$ & 3 & 0.2971 & 0.88 & \\
\hline $\mathrm{S} \times \mathrm{H}$ & 4 & 1.1441 & 5.20 & * \\
\hline $\mathrm{S} \times \mathrm{T}$ & 6 & 0.3381 & 1.65 & \\
\hline $\mathrm{H} \times \mathrm{T}$ & 6 & 0.1528 & 0.69 & \\
\hline $\mathrm{S} \times \mathrm{H} \times \mathrm{T}$ & 12 & 0.2200 & 1.08 & \\
\hline Residual & 108 & 0.2046 & & \\
\hline \multicolumn{5}{|c|}{ (d) Abundance, Monacanthidae } \\
\hline Site $(\mathrm{S})$ & 2 & 0.3831 & 0.69 & \\
\hline Habitat $(\mathrm{H})$ & 2 & 13.4537 & No test & N/A \\
\hline Time $(\mathrm{T})$ & 3 & 0.9715 & 1.75 & \\
\hline $\mathrm{S} \times \mathrm{H}$ & 4 & 1.7407 & 7.82 & ** \\
\hline $\mathrm{S} \times \mathrm{T}$ & 6 & 0.5567 & 2.94 & $*$ \\
\hline $\mathrm{H} \times \mathrm{T}$ & 6 & 0.4315 & 1.94 & \\
\hline $\mathrm{S} \times \mathrm{H} \times \mathrm{T}$ & 12 & 0.2227 & 1.18 & \\
\hline Residual & 108 & 0.1894 & & \\
\hline \multicolumn{5}{|c|}{ (e) Abundance, Gobiidae } \\
\hline Site $(\mathrm{S})$ & 2 & 2.1680 & 1338 & $* *$ \\
\hline Habitat $(\mathrm{H})$ & 2 & 12.6460 & No test & N/A \\
\hline Time $(\mathrm{T})$ & 3 & 0.1449 & 0.89 & \\
\hline $\mathrm{S} \times \mathrm{H}$ & 4 & 1.8996 & 5.34 & * \\
\hline $\mathrm{S} \times \mathrm{T}$ & 6 & 0.1621 & 1.26 & \\
\hline $\mathrm{H} \times \mathrm{T}$ & 6 & 0.1482 & 0.42 & \\
\hline $\mathrm{S} \times \mathrm{H} \times \mathrm{T}$ & 12 & 0.3557 & 2.77 & ** \\
\hline Residual & 108 & 0.1286 & & \\
\hline
\end{tabular}

There was also no systematic ranking of habitat with respect to water temperature, and differences were most likely due to the time of day that sampling was undertaken. Salinity was relatively stable throughout the study period, ranging from 34.1 to 36.1 psu across all sites, habitats and times. It is therefore considered unlikely that salinity or water temperature had any influence on the distribution of fishes. 
(a)

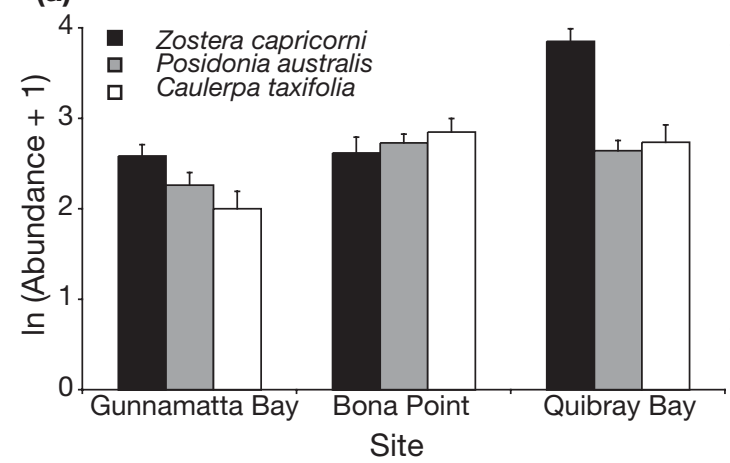

(b)

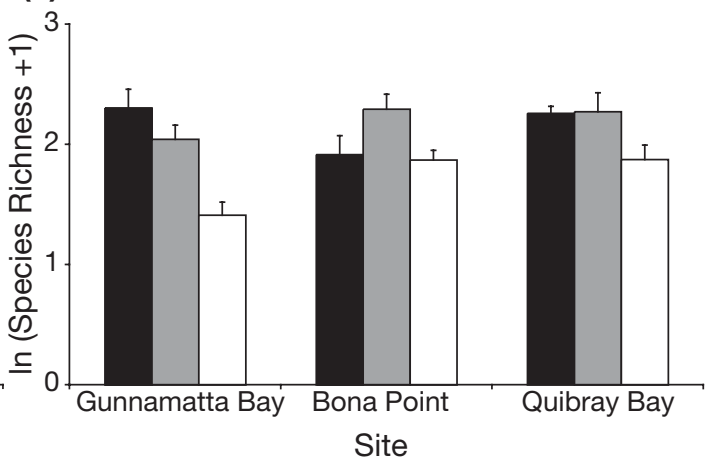

Fig. 3. Mean numbers ( \pm SE) $25 \mathrm{~m}^{-2}$ sample for (a) total abundance of fishes and (b) estimated species richness at each site for the different habitats studied. Data have been $\ln (x+1)$ transformed to reduce heterogeneity of variance
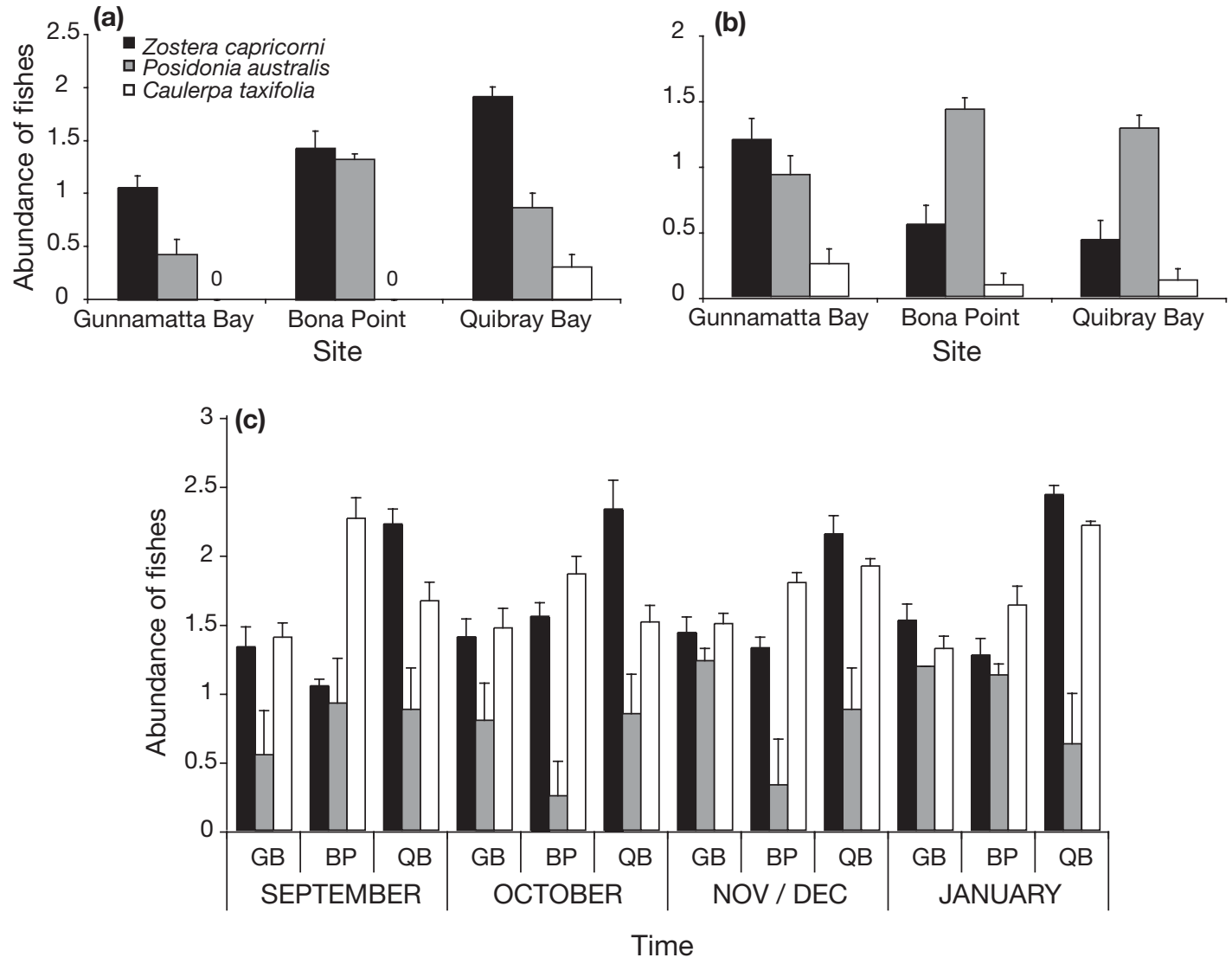

Fig. 4. Mean numbers $\left( \pm \mathrm{SE}\right.$ ) of fishes $25 \mathrm{~m}^{-2}$ sample for (a) family Syngnathidae, (b) family Monacanthidae (site and habitat) and (c) family Gobiidae (site, habitat and time). Data have been $\ln (x+1)$ transformed to reduce heterogeneity of variance

\section{DISCUSSION}

This study identified significant differences in fish assemblages among habitats dominated by Zostera capricorni, Posidonia australis or Caulerpa taxifolia. At 2 sites, the species richness of fishes and at 1 site the total abundance of fishes in $C$. taxifolia was signifi- cantly less than in the 2 seagrass habitats. Specifically, syngnathids and monacanthids were rare or absent in C. taxifolia compared to seagrasses. These results run counter to the situation in the Mediterranean, where species richness of fish assemblages is similar, though abundances of fishes are less in C. taxifolia compared to Posidonia oceanica (Francour et al. 1995), or both 
total abundance and species richness are greater in $C$. taxifolia compared to Cymodocea nodosa (Relini et al. 1998). It is possible that the results of these Mediterranean studies were confused because spatially confounded sampling designs were used, or patterns in the Mediterranean are opposite to those in New South Wales. Seagrass habitats in this study comprised similar fish assemblages to those found in previous ones in $Z$. capricorni and P. australis in south-eastern Australia (Young 1981, Middleton et al. 1984, Rotherham \& West 2002).

One possible reason for the differences in fish assemblages may be variation in structural complexity among the 3 habitats. Habitat complexity in seagrasses and other aquatic vegetation is a function of many morphological features of the plant such as leaf density and blade height, width and shape (Rooker et al. 1998). It has been demonstrated that more complex habitats are capable of supporting much greater abundances of fish because the shelter they provide is an effective aid in the avoidance of predators (Heck \& Orth 1980, Stoner \& Lewis 1985, Savino \& Stein 1993, Rooker et al. 1998). Relini et al. (2001) compared Posidonia oceania, Cymodocea nodosa and sparse and dense Caulerpa taxifolia, and found that structural complexity was important in determining the species composition of fish assemblages among habitats. Levi \& Francour (2004) also found that increases in habitat complexity due to the colonisation of previously unvegetated areas by $C$. taxifolia in the Mediterranean Sea reduced the abundances of Mullus surmuletus, a fish species usually found over bare substrate.

Diets of fishes and the availability of food in each habitat is another possible reason that may explain the differences in fish assemblages among habitats. Fish in seagrasses are known to have a variety of diets. Herbivorous and omnivorous fishes are well represented in the seagrass beds of south-eastern Australia, especially monacanthids and Girella tricuspidata (Bell \& Pollard 1989). Herbivory may be the disadvantaged in Caulerpa taxifolia habitat due to the lack of epiphytic algae growing on fronds and the toxic metabolites produced by the alga when wounded (Amade \& Lemee 1998), and indeed monacanthids and G. tricuspidata were largely absent in this habitat. Other fishes in seagrasses feed on planktonic and epifaunal crustaceans such as copepods and amphipods (Burchmore et al. 1984). Carnivorous fishes may find a reduction in food such as epifaunal and benthic invertebrates in C. taxifolia, as is the case in the Mediterranean (Boudouresque et al. 1992, Bellan-Santini et al. 1996). This may partially explain the low numbers of syngnathids in C. taxifolia; however, gobies, which are mainly carnivorous, were common in this habitat.
It is also possible that differences in fish assemblages may be due to differences in water depth between habitats. Although there were slight differences in low tide water depth, particularly between Caulerpa taxifolia and Zostera capricorni, the fact that the fish assemblages in C. taxifolia beds were more similar to those in Z. Capricorni than those in Posidonia australis indicates this is an unlikely explanation. Another possible reason for differences in fish assemblages may be an artefact of the sampling method used. The beam trawl used in the present study was biased towards smaller and slower fish (<500 mm) (Guest et al. 2003). Middleton et al. (1984) found that $P$. australis supports predominately the same species as $Z$. capricorni, although of larger body sizes, using a variety of sampling methods to target different size classes of fish. Gray \& Bell (1986) also showed that daytime sampling with a beam trawl catches slightly fewer species (30\%) and dramatically fewer individual fishes (up to $85 \%$ ) than other techniques such as night trawling and poisoning. Daytime trawling was the only feasible option available and was used consistently across all sites and habitats; however, we concede that this method does not describe the entire fish assemblage living within the sampled habitats. Several other reasons for differences in fish assemblages need to be explored further including differences in hydrodynamic factors and predation intensity among habitats.

Of the major families represented in the study, syngnathids were found to be far more abundant in seagrasses than Caulerpa taxifolia habitat. The syngnathids caught in this study were closely associated with seagrasses in previous studies in south-eastern Australia (Middleton et al. 1984, Steffe et al. 1989, Rotherham \& West 2002). It appears that these species of pipefishes are specially adapted to seagrass habitat with their colour and shape mimicking those of the seagrass blades in which they are found (Jenkins \& Wheatley 1998). Pipefish feeding behaviour, which involves them grasping the leaf blade with their tail and consuming passing copepods and amphipods, also appears to be suited to seagrass habitat (Howard \& Koehn 1985).

Monacanthids were also more abundant in seagrass beds, in particular Posidonia australis compared to Caulerpa taxifolia. Monacanthids are known to be omnivorous, with many species feeding on a diet of epiphytic algae growing on seagrass blades, invertebrates and possibly seagrass itself (Bell et al. 1978, Conacher et al. 1979, Robertson \& White 1986). Epiphytic algae were not seen growing on C. taxifolia either when it was hauled in with the net or when inspected under SCUBA. In addition, better shelter may be available to these fishes in seagrasses. Many other studies have found high abundances of mona- 
canthids in seagrass meadows and particularly among the larger leaf blades of $P$. australis (Burchmore et al. 1984, Middleton et al. 1984, Ferrell et al. 1993, Rotherham \& West 2002).

Gobies were commonly found in all 3 habitats. Previous studies show that the suite of gobiid species in this study inhabit not only seagrass but also bare sand and rocky reefs (Middleton et al. 1984, Gray et al. 1996, Jenkins \& Wheatley 1998). In this study gobies were more prevalent overall in Caulerpa taxifolia beds at both Botany Bay sites and in Zostera capricorni beds at Quibray Bay. These 2 habitats were most similar in terms of habitat complexity (leaf density, leaf height and width, but not leaf shape or colour, unpubl. data). Perhaps the adaptability of gobies to different habitats allowed them to settle in C. taxifolia and exploit the niche that is available due to the unsuitability of this habitat to other fish species (Townsend et al. 2000).

Recruits of economically important fish such as Acanthopagrus australis, Rhabdosargus sarba and Girella tricuspidata were only caught in very low numbers and predominately in Zostera capricorni in Quibray Bay. This was an unexpected result as the study coincided with the main recruitment season of juvenile fish in Botany Bay, i.e. spring and summer (Bell et al. 1988, McNeill et al. 1992, Worthington et al. 1992b, Smith \& Suthers 2000). Fish abundances in seagrasses vary both spatially and temporally (Ferrell et al. 1993), and the present study appeared to be conducted in a particularly poor year for recruitment. Given such low abundances and no definite trend between sites, it is difficult to draw strong conclusions regarding the distribution of these species between habitats. Previously, juvenile recruits of these species have been predominately distributed in Z. capricorni beds (Middleton et al. 1984, Rotherham \& West 2002). Mediterranean studies have found that sparids readily recruited to C. taxifolia (Francour et al. 1995, Relini et al. 1998).

\section{CONCLUSION}

The main finding of this study was that there were significant differences in fish assemblages among the 3 habitats of Zostera capricorni, Posidonia australis and Caulerpa taxifolia. The main differences between the assemblages of $C$. taxifolia and the 2 seagrasses were due to lower species richness in C. taxifolia due largely to an absence or rarity of fishes from the syngnathid and monacanthid families. Some of the possible reasons for these differences in fish assemblages could be differences in habitat complexity or the availability of food among the 3 habitats. Manipulative laboratory or field experiments would be useful in determining these and other likely causes of these differences. If $C$. taxifolia were to replace seagrass in New South Wales, it is possible that some fish species would decline in numbers or possibly disappear altogether. Another possibility is that once the seagrass was replaced, fish favouring this habitat would successfully switch to $C$. taxifolia beds. Further study is required to determine what the most likely outcome may be.

Acknowledgements. This study was part of an honours project funded by the Department of Environmental Sciences at the University of Technology, Sydney. Extra funding was gained through a student grant from the NSW Fisheries Scientific Committee. NSW Fisheries provided the use of the beam trawl and other equipment. Special thanks go to Dr. B. P. Kelaher for his assistance with statistical analyses. We are grateful to the 3 independent reviewers of the manuscript for their constructive comments, which helped to strengthen the paper. Also thanks to family members, friends and fellow students who made substantial contributions to field work.

\section{LITERATURE CITED}

Amade P, Lemee R (1998) Chemical defence of the Mediterranean alga Caulerpa taxifolia: variations in caulerpenyne production. Aquat Toxicol 43:287-300

Anonymous (1997) Hacking River Catchment. A Pollution Source Inventory. Hacking River Catchment Management Committee, Sydney

Bell JD, Pollard DA (1989) Ecology of fish assemblages and fisheries associated with seagrasses. In: Larkum AWD, McComb AJ, Shepard SA (eds) Biology of seagrasses. Elsevier, Amsterdam, p 565-609

Bell JD, Burchmore JJ, Pollard DA (1978) Feeding ecology of three sympatric species of leatherjackets (Pisces: Monacanthidae) from a Posidonia seagrass habitat in New South Wales. Aust J Mar Freshw Res 29:631-643

Bell JD, Steffe AS, Westoby M (1988) Location of seagrass beds in estuaries: effects on associated fish and decapods. J Exp Mar Biol Ecol 122:127-146

Bell JD, Ferrell DJ, NcNeill SE, Worthington DG (1992) Variation in assemblages of fish associated with deep and shallow margins of the seagrass Posidonia australis. Mar Biol 114:667-676

Bellan-Santini D, Arnaud PM, Bellan G, Verlaque M (1996) The influence of the introduced tropical alga Caulerpa taxifolia on the biodiversity of the Mediterranean marine biota. J Mar Biol Assoc UK 76:235-237

Boudouresque CF, Meinesz A, Verlaque M, Knoepffler-Peguy (1992) The expansion of the tropical alga Caulerpa taxifolia (Chlorophyta) in the Mediterranean. Cryptogamie Algol 13:144-145

Burchmore JJ, Pollard DA, Bell JD (1984) Community structure and trophic relationships of the fish fauna of an estuarine Posidonia australis seagrass habitat in Port Hacking, New South Wales. Aquat Bot 18:71-87

Ceccherelli G, Cinelli F (1999) Effects of Posidonia oceanica canopy on Caulerpa taxifolia size in a north-western Mediterranean bay. J Exp Mar Biol Ecol 240:19-36

Chao A (1984) Non-parametric estimation of the number of classes in a population. Scand J Stat 11:265-270

Claridge PN, Potter IC, Hardistry MW (1986) Seasonal changes in movements, abundance, size composition and 
diversity of the fish fauna of the Severn Estuary. J Mar Biol Assoc UK 66:229-258

Clarke KR (1993) Non-parametric multivariate analysis of change in community structure. Aust J Ecol 18:117-143

Clarke KR, Warwick RM (2001) Change in marine communities: an approach to statistical analysis and interpretation, Primer-E. Plymouth Marine Laboratory, Plymouth

Conacher MJ, Lanzing WJR, Larkum AWD (1979) Ecology of Botany Bay. 2. Aspects of the feeding ecology of the fanbellied leatherjacket, Monacanthus chinensis (Pisces: Monacanthidae), in Posidonia australis seagrass beds in Quibray Bay, Botany Bay, New South Wales. Aust J Mar Freshw Res 30:387-400

Ferrell DJ, Bell JD (1991) Differences among assemblages of fish associated with Zostera capricorni and bare sand over a large spatial scale. Mar Ecol Prog Ser 72:15-24

Ferrell DJ, McNeill SE, Worthington DG, Bell JD (1993) Temporal and spatial variation in the abundance of fish associated with the seagrass Posidonia australis in Southeastern Australia. Aust J Mar Freshw Res 44:881-899

Foggo A, Atrill MJ, Frost MT, Rowden AA (2003) Estimating marine species richness: An evaluation of six extrapolative techniques. Mar Ecol Prog Ser 248:15-26

Francour P, Harmelin-Vivien M, Harmelin JG, Duclerc J (1995) Impact of Caulerpa taxifolia colonisation on the littoral ichthyofauna of north-western Mediterranean Sea: preliminary results. Hydrobiologia 300/301:345-353

Glasby TM, Creese RG, Gibson PT (2005) Experimental use of salt to control the invasive marine alga Caulerpa taxifolia in New South Wales, Australia. Biol Conserv 122:573-580

Gommon MF, Glover JCM, Kuiter RH (1994) The fishes of Australia's south coast. State Print, Adelaide

Gray CA, Bell JD (1986) Consequences of two common techniques for sampling vagile macrofauna associated with the seagrass Zostera capricorni. Mar Ecol Prog Ser 28: $43-48$

Gray CA, McElligott DJ, Chick RC (1996) Intra- and interestuary differences in assemblages of fishes associated with shallow seagrass and bare sand. Mar Freshw Res 47: 723-735

Griffiths SP (2001) Diel variation in the seagrass ichthyofaunas of three intermittently open estuaries in south-eastern Australia: implications for improving fish diversity assessments. Fish Manage Ecol 8:123-140

Guest MA, Connolly RM, Loneragan NR (2003) Seine nets and beam trawls compared by day and night for sampling fish and crustaceans in shallow seagrass habitat. Fish Res 64:185-196

Heck KL Jr, Orth RJ (1980) Seagrass habitats: the roles of habitat complexity, competition and predation in structuring associated fish and motile macroinvertibrate assemblages. In: Kennedy VS (ed) Estuarine perspectives. Academic, New York, p 449-464

Henry GW, Neave P, House R (1987) A Survey of Recreational Fishing in the Port Hacking Estuary. NSW Fisheries, Sydney

Howard RK, Koehn JD (1985) Population dynamics and feeding ecology of pipefish (Sygnathidae) associated with eelgrass beds of Westernport, Victoria. Aust J Mar Freshw Res 36:361-370

Jaubert JM, Chisholm JRM, Ducrot D, Ripley HT, Roy L, Passeron-Seitre G (1999) No deleterious alterations in Posidonia beds in the bay of menton (France) eight years after Caulerpa taxifolia colonization. J Phycol 35:1113-1119

Jaubert JM, Chisholm JRM, Minghelli-Roman A, Marchioretti M, Morrow JH, Ripley HT (2003) Re-evaluation of the extent of Caulerpa taxifolia development in the northern Mediterranean using airborne spectrographic sensing. Mar Ecol Prog Ser 263:75-82

Jenkins GP, Wheatley MJ (1998) The influence of habitat structure on nearshore fish assemblages in a southern Australian embayment: comparison of shallow seagrass, reef-algal and unvegetated sand habitats, with emphasis on their importance to recruitment. J Exp Mar Biol Ecol 221:147-172

Kennish MJ (1990) Ecology of estuaries. II. Biological aspects. CRC Press, Boca Raton, FL

Kingsford MJ, Suthers IM (1996) The influence of tidal phase on patterns of ichthyoplankton abundance in the vicinity of the estuarine front, Botany Bay, Australia. Estuar Coast Shelf Sci 43:33-54

Kuiter RH (2000) The complete divers' and fisherman's guide to coastal fishes of south-eastern Australia. Gary Allen, Sydney

Levi F, Francour P (2004) Behavioral response of Mullus surmuletus to habitat modification by the invasive macroalga Caulerpa taxifolia. J Fish Biol 64:55-64

McNeill SE, Worthington DG, Ferrell DJ, Bell JD (1992) Consistently outstanding recruitment of five species of fish to a seagrass bed in Botany Bay, NSW. Aust J Ecol 17:359-365

Meinesz A, Belsher T, Thibaut T, Antolic B and 5 others (2001) The introduced green alga Caulerpa taxifolia continues to spread in the Mediterranean. Biol Invasions 3:201-210

Middleton MJ, Bell JD, Burchmore JJ, Pollard DA, Pease BC (1984) Structural differences in the fish communities of Zostera capricorni and Posidonia australis seagrass meadows in Botany Bay, New South Wales. Aquat Bot 18: 89-109

Pease BC, Herbert S (2002) Checklist of fishes from the Hacking River catchment, including the Port Hacking estuary, in central New South Wales. Wetlands 20:66-79

Pollard DA (1976) Estuaries must be protected. Aust Fish 35: $6-10$

Pollard DA (1984) A review of ecological studies on seagrass fish communities, with particular reference to recent studies in Australia. Aquat Bot 18:3-42

Potter IC, Hyndes GA (1999) Characteristics of the ichthyofaunas of southwestern Australian estuaries, including comparisons with holarctic estuaries and estuaries elsewhere in temperate Australia: a rewiew. Aust J Ecol 24:395-421

Relini G, Relini M, Torchia G (1998) Fish biodiversity in a Caulerpa taxifolia meadow in the Ligurian Sea. Ital J Zool 65:465-470

Relini M, Torchia G, Relini G (2001) Fishes of bottoms dominated by Posidonia oceanica, Cymodocea nodosa and Caulerpa taxifolia. In: Gravez V, Ruitton S, Boudouresque CF, Le Direac'h L, Meinesz A, Scabbia G, Verlaque M (eds) 4th International Workshop on Caulerpa taxifolia; Lerici, Italy, 1-2 February 1999. GIS Posidonie, Marseilles, p 246-255

Robertson CH, White RWG (1986) Feeding patterns of Mesigobius sp. Gymnapistes marmoratus, Neodax balteatus and Acanthaluteres spilomelanurus from a Tasmanian seagrass meadow. Aust J Mar Freshw Res 37:481-489

Rooker JR, Holt GJ, Holt SA (1998) Vulnerability of newly settled red drum (Sciaenops ocellatus) to predatory fish: is early life survival enhanced by seagrass meadows? Mar Biol 131:145-151

Rotherham D, West RJ (2002) Do different seagrass species support distinct fish communities in south-eastern Australia? Fish Manage Ecol 9:235-248

Savino JF, Stein RA (1993) Behavioural interactions between fish predators and their prey: effects of plant density. Anim Behav 37:311-321 
Schaffelke B, Murphy N, Uthicke S (2002) Using genetic techniques to investigate the sources of the invasive alga Caulerpa taxifolia in three new locations in Australia. Mar Pollut Bull 44:204-210

Smith KA, Suthers IM (2000) Consistent timing of juvenile fish recruitment to seagrass beds within two Sydney estuaries. Mar Freshw Res 51:765-776

Steffe AS, Westoby M, Bell JD (1989) Habitat selection and diet in two species of pipefish from seagrass: sex differences. Mar Ecol Prog Ser 55:23-30

Stoner AW, Lewis FGI (1985) The influence of quantitative and qualitative aspects of habitat complexity in tropical seagrass meadows. J Exp Mar Biol Ecol 94:19-40

Townsend CR, Harper JL, Begon M (2000) Interspecific competition. In: Townsend CR, Harper JL, Begon M (eds)

Editorial responsibility: Otto Kinne (Editor-in-Chief), Oldendorf/Luhe, Germany
Essentials of ecology. Blackwell, Malden, MA, p 203-236

West RJ, Thorogood CA, Walford TR, Williams RJ (1985) An estuarine inventory for New South Wales, Australia. Department of Agriculture New South Wales, Sydney

Worthington DG, Ferrell DJ, McNeill SE, Bell JD (1992a) Effects of the shoot density of seagrass on fish and decapods: are correlations evident over larger spatial scales? Mar Biol 116:139-146

Worthington DG, Ferrell DJ, McNeill SE, Bell JD (1992b) Growth of four species of juvenile fish associated with the seagrass, Zostera capricorni in Botany Bay, New South Wales. Aust J Mar Freshw Res 43:1189-1198

Young PC (1981) Temporal changes in the vagile epibenthic fauna of two seagrass meadows (Zostera capricorni and Posidonia australis). Mar Ecol Prog Ser 5:91-102

Submitted: December 22, 2004; Accepted: October 6, 2005 Proofs received from author(s): March 17, 2006 